\title{
Squamous Cell Carcinoma Arising from Aparaurethral Region
}

\author{
Kyosuke Matsuzaki ${ }^{1}$, Takahiro Yoshida', Yasuyuki Kobayashi' ${ }^{1}$, Ken Takeda', \\ Yasuyuki Arai', Masashi Nakayama1, Ken-ichi Kakimoto1, \\ Yasuhiko Tomita ${ }^{2}$, Kazuo Nishimura ${ }^{1}$ \\ ${ }^{1}$ Department of Urology, Osaka Medical Center for Cancer and Cardiovascular Diseases, Osaka, Japan \\ ${ }^{2}$ Department of Pathology, Osaka Medical Center for Cancer and Cardiovascular Diseases, Osaka, Japan \\ Email: nisimura-ka2@mc.pref.osaka.jp
}

Received 18 March 2016; accepted 23 April 2016; published 26 April 2016

Copyright (C) 2016 by authors and Scientific Research Publishing Inc.

This work is licensed under the Creative Commons Attribution International License (CC BY). http://creativecommons.org/licenses/by/4.0/

(c) (7) Open Access

\section{Abstract}

A 69-year-old man was referred to our hospital with dysuria and gross hematuria associated with a rapidly growing perineal mass. Serum squamous cell carcinoma (SCC) antigen level was high (2.4 $\mathrm{ng} / \mathrm{mL}$ ). Magnetic resonance imaging examination revealed a tumor posterior and inferior to the pendular urethra with a cystic lesion inside. Tumor resection together with total penectomy was performed. Pathohistological findings revealed well-differentiated SCC arising from the paraurethral region. The patient remained free of disease without adjuvant therapy at 70 months after surgery. To our knowledge, this is the second reported case of paraurethral SCC.

\section{Keywords}

\section{Paraurethral, Squamous Cell Carcinoma, Perineal Mass}

\section{Introduction}

Primary urethral carcinoma represents a rare malignancy accounting for $<1 \%$ of genitourinary cancers. As for histological types, squamous cell carcinoma (SCC) is most common among both genders but adenocarcinomas are noted in $15 \%$ - 35\% of cases among women [1]. As female urethral diverticula are considered to be associated with adenocarcinoma [2], paraurethral adenocarcinoma is relatively well reported [3]-[5]. Meanwhile, paraurethral SCC is extremely rare. We report a case of paraurethral SCC presenting as a rapidly growing perineal mass.

\section{Case Report}

A 69-year-old man with no past medical history was referred to our hospital complaining of dysuria and gross

How to cite this paper: Matsuzaki, K., Yoshida, T., Kobayashi, Y., Takeda, K., Arai, Y., Nakayama, M., Kakimoto, K., Tomita, Y. and Nishimura, K. (2016) Squamous Cell Carcinoma Arising from Aparaurethral Region. Case Reports in Clinical Medicine, 5, 154-157. http://dx.doi.org/10.4236/crcm.2016.54029 
hematuria associated with a rapidly growing perineal mass. Physical examination revealed a stiff mass in the perineum. Digital rectal examination showed no abnormal findings. Serum laboratory data showed inflammatory findings, and among several tumor markers measured, only the SCC antigen level was slightly high at 2.4 $\mathrm{ng} / \mathrm{mL}$. Magnetic resonance imaging (MRI) examination revealed a tumor $(45 \times 52 \times 55 \mathrm{~mm})$ posterior and inferior to the pendular urethra with a cystic lesion inside, which was invading the corpusspongiosum (Figure 1). Urine cytology was positive, suggesting SCC. Cystourethroscopy revealed a constriction at the posterior urethra but no clear finding of urethral tumor. The patient then developed urinary retention and for which percutaneous cystostomy was performed. Percutaneous tumor needle biopsy showed SCC. Tumor resection and total penectomy were then performed. The bilateral testes were preserved. The excised tumor appeared as a solid and grayish mass, approximately $5 \mathrm{~cm}$ in size, with a yellowish granulomatous lesion next to the urethra (Figure 2). Pathohistological findings revealed well-differentiated SCC arising from the paraurethral region (Figure 3(A)). Neither the scrotal skin nor the urethra was directly infiltrated by the tumor (Figure 3(B), Figure 3(C)), while the urethral constriction was involved with the granulomatous lesion which was determined to be inflammation associated with tumor spillage (Figure 3(D)). The surgical margin was negative. Postoperatively, no additional treatment was administered. As of 70 months after surgery, the SCC antigen level was normal, and the patient remained free of disease.

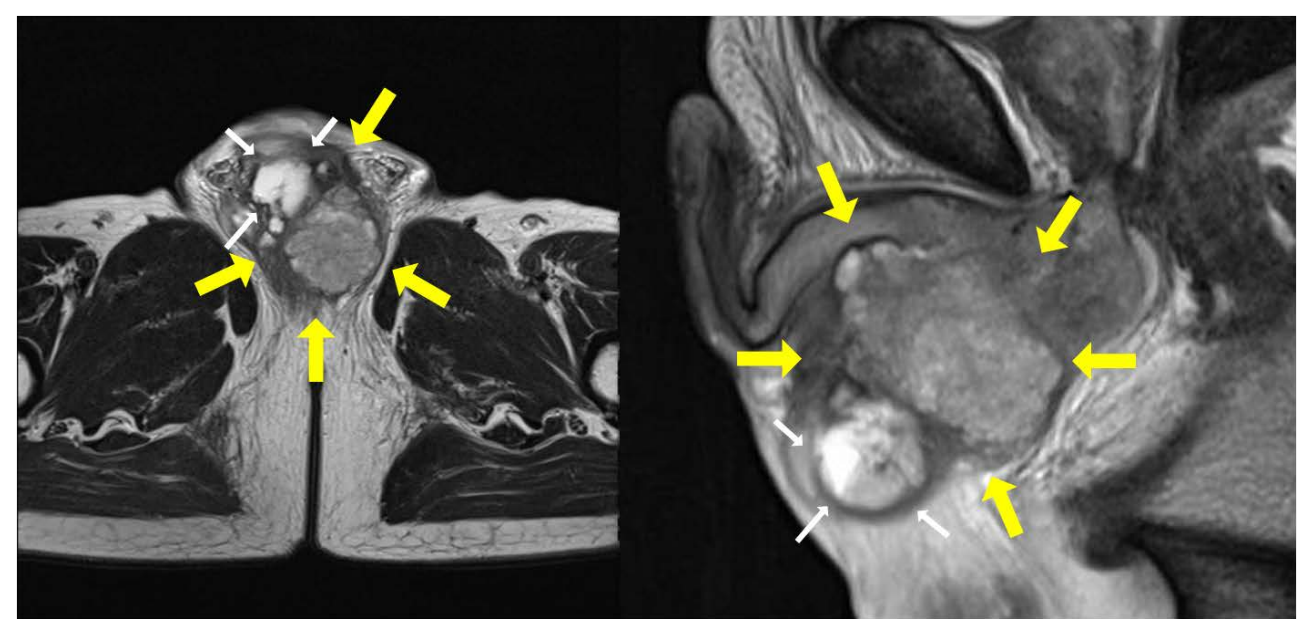

Figure 1. Magnetic resonance imaging revealed a tumor posterior and inferior to the pendular urethra. Large arrows indicate tumor and small arrows indicate the cystic lesion. Left panel: transverse plane. Right panel: sagittal plane.

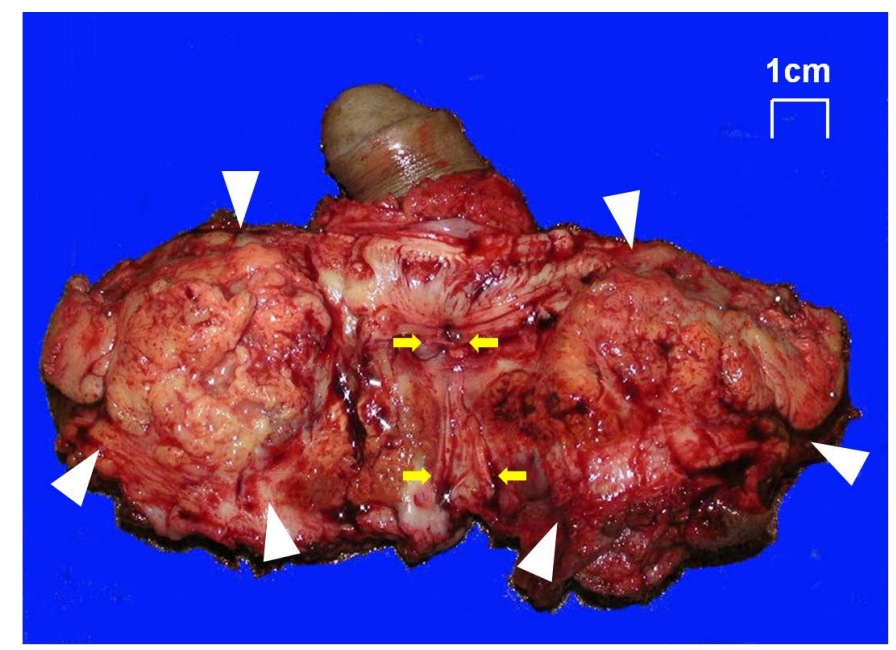

Figure 2. Macroscopic appearance of the tumor. The tumor is solid and grayish, apart from the urethra. Arrowheads indicate tumor and arrows indicate urethra. 


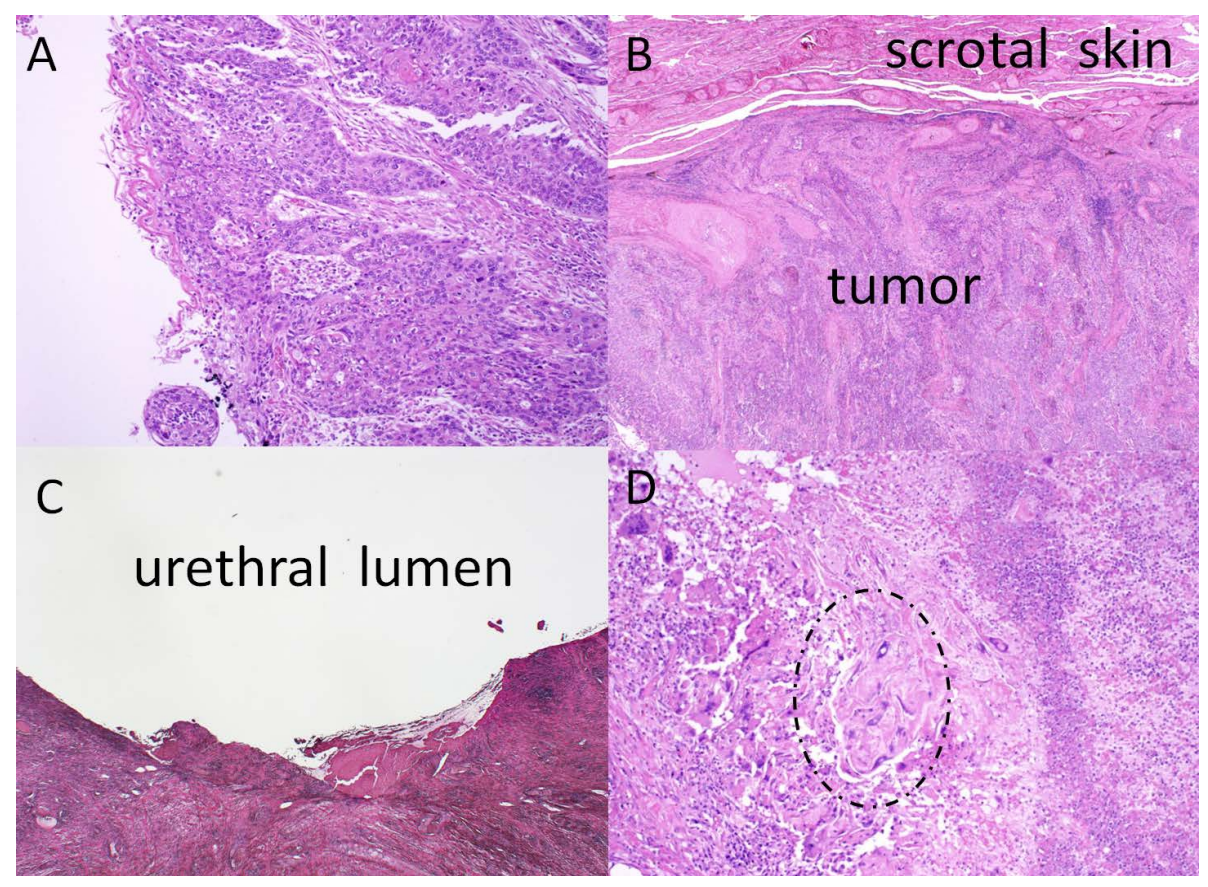

Figure 3. Microscopic appearance of the tumor. (A) Histology of the tumor shows well-differentiated squamous cell carcinoma (hematoxylin and eosin [H\&E] stain, $\times 100)$. Neither scrotal skin (B) nor the urethral lumen (C) was invaded directly by the tumor (H\&E stain, $\times 100$ ). D. Granulomatous inflammatory lesion next to the urethra with tumor cell spillage, as outlined by the dashed line (H\&E stain, $\times 400)$.

\section{Discussion}

Although female paraurethral adenocarcinoma is relatively well reported [3]-[5], paraurethral SCC is extremely rare in both genders. To the best of our knowledge, only one case has been reported previously by Barua et al., who reported a 58-year-old man complaining chiefly of hematuria and a firm mass [6]. The tumor was located posterior and inferior to the bladder with a cyst inside. Histological findings revealed SCC arising within a paraurethral cyst. Their patient died 12 months after surgical removal of the tumor.

In the present case, a cystic lesion was identified within the tumor by MRI (Figure 1), but this lesion was collapsed at the time of the percutaneous biopsy by chance. Then the cyst was shrunk and compressed by the tumor in the resected specimen. There was a granulomatous inflammatory lesion next to the urethra with tumor cell spillage (Figure 3(D)). This suggests that tumor cells were spilling into the necrotic tissue due to inflammatory change, which resulted in the positive urine cytology. Neither the urethra nor the skin, which is a common primary site of SCC among perineal organs, was directly involved.

There are several hypotheses as to the origin of SCC, including ectopic squamous cells in the paraurethral tissue, the urethral glands such as Cowper's gland or Skene's glands, and a paraurethral cyst as reported previously [6]. Whereas the paraurethral cystic lesion identified on MRI is considered as a possible origin in the present case (Figure 1), the histological examination could not confirm this due to the shrinkage of the cyst by the previous biopsy.

Clinical signs of paraurethral carcinoma are assumed to be similar with urethral carcinoma [3]-[7]. Obstructive or irritative symptoms and hematuria are common modes of presentation of urethral carcinoma [1]. Urethral obstruction is evaluated by cystourethroscopy with cold cup biopsy but percutaneous needle biopsy may be required depending on the tumor location. MRI is a highly effective method to image the primary tumor to establish the loco-regional extent of disease at diagnosis. [1] In our case, the tumor invaded the corpus spongiosum without regional nodal involvement or distant metastasis. Since the surgical margin was negative, no additional treatment was added. Similar to urethral carcinoma, radiation therapy can be used as an adjuvant treatment if a surgical margin is positive [1]. No standard systemic therapy has been established due to the rarity of paraurethral carcinoma. In a retrospective study of 44 patients with advanced urethral carcinoma, cisplatin-based chemo- 
therapy according to the primary histology was reported [8]. The median overall survival from chemotherapy initiation was 25.6 months. Although most urethral carcinomas respond well to cisplatin-based chemotherapy, further studies are needed to define the role of chemotherapy [1]. Since treatment modality other than surgery has not been reported for paraurethral SCC, complete resection should be tried to achieve long-term survival.

\section{Conclusion}

This report presented a case of paraurethral SCC in a 69-year-old man who achieved a long-term disease-free survival after surgery. Given a rarity of this disease, timely diagnosis and complete resection of tumor are critical for disease-free survival.

\section{Informed Consents}

Informed consent was obtained from the patient.

\section{Conflict of Interest}

None.

\section{References}

[1] Dayyani, F., Hoffman, K., Eifel, P., Guo, C., Vikram, R., et al. (2014) Management of Advanced Primary Urethral Carcinomas. BJU International, 114, 25-31. http://dx.doi.org/10.1111/bju.12630

[2] Thomas, A.A., Rackley, R.R., Lee, U., Goldman, H.B., Vasavada, S.P., et al. (2008) Urethral Diverticula in 90 Female Patients: A Study with Emphasis on Neoplastic Alterations. The Journal of Urology, 180, 2463-2467. http://dx.doi.org/10.1016/j.juro.2008.08.040

[3] Nagano, M., Hasui, Y., Ide, H., Itoi, T., Takehara, T., et al. (2002) Primary Adenocarcinoma Arising from a Paraurethral Cyst in a Female Patient. Urology International, 69, 244-246. http://dx.doi.org/10.1159/000063937

[4] Massari, F., Ciccarese, C., Modena, A., Maines, F., Segala, D., et al. (2014) Adenocarcinoma of the Paraurethral Glands: A Case Report. Histology and Histopathology, 29, 1295-1303.

[5] Islam, A.H., Kato, H., Hayama, M., Kobayashi, S., Ota, H., et al. (2001) Adenocarcinoma of Female Paraurethral Duct Showing Neuroendocrine Differentiation. Urology, 58, 1058. http://dx.doi.org/10.1016/S0090-4295(01)01437-6

[6] Barua, J.M., Bates, A.W. and Baithun, S.I. (1997) Squamous Cell Carcinoma Arising in a Para-Urethral Cyst. British Journal of Urology, 80, 158-159. http://dx.doi.org/10.1046/j.1464-410X.1997.00283.X

[7] Syvänen, K.T., Taimen, P., Salminen, A., Kuusisto, K. and Boström, P.J. (2014) Bulbourethral Gland Adenocarcinoma in a 25-Year-Old Man without Comorbidities: Radical Resection of Proximal Urethrae with Mitrofanoff-Type Appendicovesicostomy. Scandinavian Journal of Urology, 48, 405-409. http://dx.doi.org/10.3109/21681805.2013.852622

[8] Dayyani, F., Pettaway, C.A., Kamat, A.M., Munsell, M.F., Sircar, K. and Pagliaro, L.C. (2013) Retrospective Analysis of Survival Outcomes and the Role of Cisplatin-Based Chemotherapy in Patients with Urethral Carcinomas Referred to Medical Oncologists. Urologic Oncology, 31, 1171-1177. http://dx.doi.org/10.1016/j.urolonc.2012.01.011 